\title{
On a generalized reflection principle in $\mathbb{C}^{2}$
}

\author{
Peter Ebenfelt and Xiaojun Huang*
}

\section{Introduction}

The classical Schwarz reflection principle in the complex plane $\mathbb{C}$ can be formulated as follows. Assume that $F U \rightarrow \mathbb{C}$, where $U$ is an open subset of $\mathbb{C}$, is a holomorphic mapping which extends continuously to a real-analytic curve $M$ contained in the boundary of $U$. If $F$ maps $M$ into another real-analytic curve $M^{\prime} \subset \mathbb{C}$, then $F$ extends holomorphically to an open neighborhood of $M$. In higher dimensions the situation is much more complicated and the analogous statement fails in general as can be seen by easy examples. To find conditions under which a reflection principle holds is an interesting and challenging problem, which has been addressed by many mathematicians. We mention here only the papers [BJT], [BBR], [BR1], [BR2], [DF], [BHR], [Hu1], [HMM] and refer the interested reader to the book [BE] and the recent survey article [Hu2] for a more detailed account of the history. However, we would like to point out that the following conjecture is still open in dimensions higher than three. Let $M, M^{\prime} \subset \mathbb{C}^{n+1}$ be real-analytic hypersurfaces through the origin and $U \subset \mathbb{C}^{n+1}$ an open set containing $M$ in its boundary. Assume that neither $M$ nor $M^{\prime}$ contains a nontrivial complex analytic variety through 0 . Then any holomorphic mapping $F U \rightarrow \mathbb{C}^{n+1}$ which extends continuously to $M$, sending $M$ into $M^{\prime}$ and 0 to 0 extends holomorphically to an open neighborhood of 0 . The second author [Hu1] proved this conjecture in $\mathbb{C}^{2}$.

If $M^{\prime}$ contains a nontrivial complex analytic variety through 0 , then a local reflection principle cannot hold at 0 unless additional conditions (which exclude the possibility that all of $M$ is mapped into the variety) are imposed on the mapping $f$. In [HMM] a reflection principle was proved in a situation (in $\mathbb{C}^{n+1}$ ) where $M$ and $M^{\prime}$ are allowed to contain a complex analytic hypersurface through 0 and $F$ extends as a suitably nondegenerate $C^{1}$ (continuously differentiable) mapping of $M$ to $M^{\prime}$. In the present paper, we consider this situation in $\mathbb{C}^{2}$ and by making use of the more complete geometric picture of real hypersurfaces in $\mathbb{C}^{2}$, we are able to show that a

\footnotetext{
${ }^{*}$ P. Ebenfelt was supported in part by a Swedish NFR; X. Huang was supported in part by NSF-9970439 and the Rutgers Research Council
}

Complex Analysis and Geometry

Ohio State Univ. Math. Res. Inst. Publ. 9

(C) Walter de Gruyter 2001 
reflection principle holds for similarly nondegenerate $F$ which merely extend as continuous mappings. The main novelty here is Lemma 2.4 below which establishes the continuity of the derivative of the mapping along a certain vector field. Another crucial result is Proposition 3.1, which was obtained in [HMM] with the additional assumption of Hölder continuity.

As a consequence, by using the result in [Hu1], we obtain the following; recall that a connected real-analytic hypersurface in $\mathbb{C}^{n+1}$ is called Levi flat if it is locally biholomorphic, near every point, to a real hyperplane, i.e., a $2 n+1$ dimensional linear subspace of $\mathbb{R}^{2 n+1} \cong \mathbb{C}^{n+1}$.

Theorem 1. Let $M, M^{\prime} \subset \mathbb{C}^{2}$ be real-analytic Levi non-flat hypersurfaces and $D \subset$ $\mathbb{C}^{2}$ a domain (connected open set) with $M$ in its boundary. Assume that $F D \rightarrow \mathbb{C}^{2}$ is a holomorphic mapping which extends continuously to $M$ and sends $M$ into $M^{\prime}$. If

(i) $F(D)$ is not contained in a 1-dimensional complex analytic variety, and

(ii) F restricted to any nontrivial complex analytic variety contained in $M$ is not constant,

then $F$ extends holomorphically to an open neighborhood of $M$.

As a corollary, we obtain the following result.

Corollary 1. Let $M, M^{\prime} \subset \mathbb{C}^{2}$ be real-analytic Levi non-flat hypersurfaces and $D \subset$ $\mathbb{C}^{2}$ a domain with $M$ in its boundary. Assume that $F D \rightarrow \mathbb{C}^{2}$ is a holomorphic mapping which extends continuously to $M$. If $F M \rightarrow M^{\prime}$ is a finite-to-one mapping, then $F$ extends holomorphically to an open neighborhood of $M$.

Theorem 1 will be a consequence of the result in [Hu1] and the following, which is the main contribution of this paper.

Theorem 2. Let $M \subset \mathbb{C}^{2}$ be a real-analytic Levi nonflat hypersurface which contains a nontrivial complex analytic variety $E$ through 0 . Let $D \subset \mathbb{C}^{2}$ be an open connected set which contains $M$ in its boundary. Assume that $F D \rightarrow \mathbb{C}^{2}$ is a holomorphic mapping which extends continuously to $M$ and sends $M$ into another real-analytic hypersurface $M^{\prime} \subset \mathbb{C}^{2}$. If

(i) $F(D)$ is not contained in a 1-dimensional complex analytic variety, and

(ii) $\left.F\right|_{E}$ is not constant,

then $F$ extends holomorphically to an open neighborhood of 0 in $\mathbb{C}^{2}$.

Remark 1. As will become apparent from the proof of Theorem 2, condition (i) is in fact equivalent to $J_{F} \not \equiv 0$ in $D$, where $J_{F}$ denotes the Jacobian determinant of $F$ i.e., $J_{F}:=\operatorname{det}(\partial F / \partial Z)$. Clearly, (i) is implied by the latter. The converse is a consequence 
of the fact that $F(M) \subset M^{\prime}$. (Indeed, (i) does not imply $J_{F} \not \equiv 0$ in $D$ without this condition.) We also mention that the presence of a complex analytic curve in $M$ enables us to get the single-valued holomorphic extension directly. This is in contrast with the proof of the holomorphic extension in the finite type case ([Hu1]), where one has to split the proof in two separated steps: Step (a): get the multiple-valued extension (or get the correspondence extension in some literature), assuming the result in the following Step (b); Step (b): Prove the single-valued property assuming the map already extends as a multiple-valued analytic maps. (This step is usually called the study of the regularity of correspondences (see [Hu3])).

We would like to point out that if $F$ in Theorem 2 is assumed to extend as a $C^{1}$ mapping to $M$, then the conclusion follows from the result in [HMM]. If $F$ extends as a $C^{\infty}$ mapping and $J_{F}$ does not vanish to infinite order at 0 (which implies (i) in Theorem 2) then, in view of the reflection principle in [BR2], condition (ii) in Theorem 2 is redundant. However, if only a finite degree of smoothness is required then condition (ii) is needed as the following example illustrates.

Example 1 ([HMM]). Let $M, M^{\prime} \subset \mathbb{C}^{2}$ be given by

$$
\begin{aligned}
M & =\left\{(z, w) \in \mathbb{C}^{2}: \operatorname{Im} w=(\operatorname{Re} w)^{2}|w|^{2 k^{2}+k}|z|^{2 k}\right\}, \\
M^{\prime} & =\left\{(z, w) \in \mathbb{C}^{2}: \operatorname{Im} w=(\operatorname{Re} w)^{2}|z|^{2 k}\right\} .
\end{aligned}
$$

Consider

$$
F(z, w)=\left(z w^{k+1 / 2}, w\right)
$$

where $k$ is even. Then $F$ is a holomorphic mapping $D \rightarrow \mathbb{C}^{2}$, where

$$
D=\left\{\left(z, w \operatorname{Im} w>(\operatorname{Re} w)^{2}|w|^{2 k^{2}+k}|z|^{2 k}\right\},\right.
$$

which extends as a $C^{k, 1 / 2}$ mapping $M \rightarrow M^{\prime}$ but not as a holomorphic mapping in any neighborhood of 0 in $\mathbb{C}^{2}$. Observe that $J_{F}$ vanishes to finite order at 0 and, hence, $F$ satisfies condition (i) of Theorem 2 . However, $F$ does not satisfy condition (ii), since $F^{-1}(0)$ is the complex hyperplane $\{w=0\} \subset M$.

We would like to conclude the introduction with an open problem. Recall that a real hypersurface $M \subset \mathbb{C}^{n+1}$ inherits a $\mathrm{CR}$ structure $T^{0,1} M \subset \mathbb{C} T M$ from the ambient space; the subspace $T_{p}^{0,1} M$ is the $n$-dimensional complex vector space consisting of those $X \in T^{0,1} p \mathbb{C}^{n+1}$ which are also in tangent to $M$. Sections of $T^{0,1} M$ are called CR vector fields. A locally integrable function on $M$ is called a CR function if it is annihilated by all CR vector fields, and a mapping $F M \rightarrow M^{\prime} \subset \mathbb{C}^{n+1}$ is called CR if its components $F_{j}$ are CR functions. (The reader is referred to [BE] for an introduction to CR geometry and basic results.) An important result is the following. All continuous CR functions defined in a neighborhood of 0 in $M$ arise as boundary values of holomorphic functions defined on one side of $M$ if and only if $M$ does not contain a complex analytic hypersurface though 0 (see [Tr]; see also [BT1] 
and [Tu]). Hence, if $M$ and $M^{\prime}$ are as in Theorem 2, then the class of continuous CR mappings $F M \rightarrow M^{\prime}$ is potentially larger than the class of boundary values of holomorpic mappings considered in Theorem 2 and one may ask: Does the conclusion of Theorem 2 also hold for the class of continuous CR mappings (which would then imply that the two classes coincide)? The answer is negative as is demonstrated by the following example.

Example 2. Let $M, M^{\prime} \subset \mathbb{C}^{2}$ be given by

$$
\begin{aligned}
M & =\left\{(z, w) \in \mathbb{C}^{2} \operatorname{Im} w=(\operatorname{Re} w)|z|^{2}\right\} \\
M^{\prime} & =\left\{(z, w) \in \mathbb{C}^{2} \operatorname{Im} w=(\operatorname{Re} w) h_{k}(z, \bar{z})\right\} .
\end{aligned}
$$

where $h_{k}$ is defined as follows. Define the finite sequences $\left\{a_{k}\right\}$ and $\left\{b_{k}\right\}$ by

$$
\begin{gathered}
\phi_{k}(s, t)=\operatorname{Re}(s+i t)^{k}=s^{n}+\sum_{j=0}^{k-1} a_{j} s^{j} t^{k-j} \\
\psi_{k}(s, t)=\operatorname{Im}(s+i t)^{k}=\sum_{j=0}^{k-1} b_{j} s^{j} t^{k-j}
\end{gathered}
$$

and set

$$
h_{k}:=\frac{\sum_{j=0}^{k-1} b_{j}|z|^{2(k-j)}}{1+\sum_{j=0}^{k-1} a_{j}|z|^{2(k-j)}} .
$$

Let $F=(z, g)$ with $g=-w^{k}$ for $s \leq 0$ and $g=w^{k}$ for $s>0$. Then $F$ is a finite to one $C^{k-1}$-differentiable CR mapping from $M$ into $M^{\prime}$, which does not extend holomorphically to any neighborhood of 0 . Observe that $F$ does not extend holomorphically to any side of $M$, nor is it $C^{k}$-differentiable over $M$. Related to this example, we mention a result proved in [BHR] for algebraic CR manifolds, which, in particular, implies that if the degree of smoothness of the CR mapping $F$ is sufficiently high relative to the degree of the target manifold $M^{\prime}$, then $F$ does extend holomorphically (indeed, algebraically) to a neighborhood of 0 .

Thus, the assumption that $F$ in Theorem 2 extends holomorphically to one side of $M$ cannot be removed. However, the authors have some hope that the following is true.

Conjecture. Let $M, M^{\prime} \subset \mathbb{C}^{2}$ be real-analytic Levi nonflat hypersurfaces. Then any $C^{\infty}$-smooth CR (local) diffeomorphism $F M \rightarrow M^{\prime}$ extends holomorphically to an open neighborhood of $M$ in $\mathbb{C}^{2}$.

Note that $F$ in the conjecture, by known results mentioned above, extends holomorphically to an open neighborhood in $\mathbb{C}^{2}$ of a dense open subset of $M$, namely the 
set of points through which there are no complex analytic hypersurfaces contained in $M$. For future reference, we recall that a real hypersurface which does not contain a complex analytic hypersurface through a point $p \in M$ is said to be minimal at $p$. For a real-analytic hypersurface, minimality at a point is equivalent to being of finite type in the sense of Kohn and Bloom-Graham at this point. We also mention that if $M$ and $M^{\prime}$ in addition are assumed to by algebraic in the conjecture, then the conclusion follows from the results in [BHR].

\section{Geometry of the Segre varieties near an infinite type point}

For the proof of the Theorem 2, we will use some ideas in [HMM]. However there would be a serious difficulty if we try to directly modify it to the case of continuous mappings. This is because the argument in [HMM] needs to deal with the quotient of certain distributions coming from the differential of the map. In this paper, we will find a way to deal with this in the case of complex two-space by employing geometric information of the Segre invariant family near an infinite type point. The purpose of this section is to make a preparation towards such an effort.

Let $M$ be a Levi non-flat real analytic hypersurface in $\mathbb{C}^{2}$ with $0 \in M$ an infinite type point. Then, after a holomorphic change of variables, we can assume that $M$ near 0 is defined by an equation of the form: $t=s^{k} h(z, \bar{z}, s)$, where $k \in \mathbb{Z}^{+}$, $h(z, 0, s)=h(0, \bar{z}, s) \equiv 0$ and $h(z, \bar{z}, 0) \neq 0$. Also, in what follows, we use $(z, w)$, with $w=s+i t$, for the coordinates of $\mathbb{C}^{2}$. Write $m(0)$ for the lowest degree of the non-vanishing terms in the Taylor expansion of $h(z, \bar{z}, 0)$ near 0 . It is known (see [Me]) that $k, m(0)$ are holomorphic invariant of $M$. Moreover, in such a coordinates system, $M$ contains the complex curve $E=\{w=0\}$ and for each $p_{0}=\left(z_{0}, 0\right)$ near 0 (which in what follows will be abbreviated $p_{0} \approx 0 M$ has the same $k$-value, but $m\left(p_{0}\right)$ may well depend on $p_{0}$.

Let $u(z, w)=t-s^{k} h(z, \bar{z}, s)$. Then the Levi determinant of $M$ associated to the defining function $u$ is given by

$$
J_{u}:=\operatorname{det}\left(\begin{array}{ccc}
0 & u_{z}^{\prime} & u_{w}^{\prime} \\
u_{\bar{z}}^{\prime} & u_{z \bar{z}}^{\prime \prime} & u_{w \bar{z}}^{\prime \prime} \\
u_{\bar{w}}^{\prime} & u_{z \bar{w}}^{\prime \prime} & u_{w \bar{w}}^{\prime \prime}
\end{array}\right) .
$$

A direct computation shows that $J_{u}(z, \bar{z}, s)=s^{k} \Delta(z, \bar{z}, s)$ and the lowest degree of the non-vanishing terms in the Taylor expansion of $\Delta(z, \bar{z}, 0)$ near 0 is $m(0)-2$. Let $E_{0}$ be the zero set of $\Delta$ in $E$, which is a thin set in $E$. Then it is clear that for each $p_{0} \in E \backslash E_{0}, m\left(p_{0}\right)=2$. Hence, we have

Lemma 2.1. For each $p_{0} \in E \backslash E_{0}$, there are holomorphic coordinates $(z, w)$, vanishing at $p_{0}$, in which $M$ can be defined, near $p_{0}=(0,0)$, by an equation of the form $t=s^{k} h(z, \bar{z}, s)$, where $h(z, \bar{z}, 0)= \pm|z|^{2}+o\left(|z|^{2}\right)$ and $h(z, 0, s)=h(0, \bar{z}, s) \equiv 0$. 
Now assume that 0 is an infinite type point of $M$ with $m(0)=2$. We need some basic property of Segre varieties near such a point. To this aim, we set up the following notation. The reader is referred to [Hu1] for a more detailed explanation of the notation and definitions.

Choose two sufficiently small balls $\mathcal{P}, \widetilde{\mathcal{P}}$ centered at 0 with $\mathcal{P} \subset \subset \widetilde{\mathcal{P}}$. Making the radius of $\mathcal{P}$ much smaller than that of $\widetilde{\mathcal{P}}$, we can assume that for each $p \in \mathcal{P}$, the Segre variety $Q_{p}$ of $M$ associated to $p$ is a closed complex hypersurface in $\widetilde{\mathcal{P}}$. Also, there is a side-reversing (with respect to $M$ ) diffeomorphic map from $\mathcal{P}$ into its image which is contained in $\widetilde{\mathcal{P}}$, called the conjugate map $\mathcal{R}$, such that $\mathcal{R}(p) \in Q_{p}$ for each $p \in \mathcal{P}$. Also, $\left.\mathcal{R}\right|_{M}=\mathrm{id}, \mathcal{R}(p)$ and $p$ always stay on different sides of $M$ for $p \notin M$. For the system chosen above, we say that a point $p \in \mathcal{P}$ stays in the positive side of $M$ if the connected component of $Q_{p} \backslash M$ containing $\mathcal{R}(p)$ is compact in $\widetilde{\mathcal{P}}$. In this case, we denote such a component by $Q_{p}^{c}$.

For each $p=(z, w) \in M \cap \mathcal{P}$ and a positive number $\epsilon$, write $N_{\epsilon, p}^{ \pm}=\{(z, w) \pm$ $(0, i \tau \epsilon): 0<\tau \leq 1\}$, respectively, and $N_{\epsilon, p}=N_{\epsilon, p}^{+} \cup N_{\epsilon, p}^{-} \cup\{p\}$.

Lemma 2.2. Assume $\mathcal{P}$ is sufficiently small and $\delta$ is a sufficiently small positive number. For any $p_{0}=\left(z_{0}, w_{0}\right) \in \mathscr{P} \cap M$ with $w_{0}=s_{0}+i t_{0}$, consider the set $N_{\epsilon, p_{0}}^{ \pm}$with $\epsilon<\delta\left|s_{0}\right|^{k}$. Then any point in $N_{\epsilon, p_{0}}^{-}$stays in the positive side of $M$ in the following cases: (I) $h(z, \bar{z}, 0)=|z|^{2}+o\left(|z|^{2}\right), s_{0}>0$; (II) $h(z, \bar{z}, 0)=|z|^{2}+o\left(|z|^{2}\right), s_{0}<0$, and $k$ is even; (III) $h(z, \bar{z}, 0)=-|z|^{2}+o\left(|z|^{2}\right), s_{0}<0$ and $k$ is odd. In all other situations, $N_{\epsilon, p_{0}}^{+}$must stay in the positive side of $M$.

Proof. In the following we fix a small $\widetilde{\mathcal{P}}$ but reserve the right to shrink $\mathcal{P}$. We first assume that $h(z, \bar{z}, 0)=|z|^{2}+o\left(|z|^{2}\right)$, and let $p_{0}=\left(z_{0}, s_{0}+i t_{0}\right) \in \mathcal{P}$ with $s_{0}>0$. We will verify that any point in $N_{\epsilon, p_{0}}^{-}$stays in the positive side of $M$ in the sense defined above, whenever $\epsilon<\delta s_{0}^{k}$ with $\delta>0$ a sufficiently small number (but independent of the particular choice of $\left.p_{0}\right)$. Let $p_{\tau}=\left(z_{0}, w_{\tau}\right)=\left(z_{0}, s_{0}+i t_{0}-i \tau \epsilon\right) \in N_{\epsilon, p_{0}}^{-}$. Then

$$
Q_{p_{\tau}}=\left\{(z, w) \in \widetilde{\mathcal{P}}: \frac{w-\overline{w_{\tau}}}{2 i}=\left(\frac{w+\overline{w_{\tau}}}{2}\right)^{k} h\left(z, \overline{z_{0}}, \frac{w+\overline{w_{\tau}}}{2}\right)\right\} .
$$

Recall the notation $u=-t+s^{k} h(z, \bar{z}, s)$. It is then clear that $M$ is strongly pseudoconvex near $p_{\tau}$ towards the side defined by $u(z, w)<0$. Now, by a fundamental observation of Webster ([We2] or Lemma 2.2 of [Hu1]), when $\tau \ll 1, p_{\tau}$ stays in the positive side of $M$ and the diameter of $Q_{p_{\tau}}^{c}(\subset\{u<0\})$ is about $\sqrt{\tau}$. Notice that $\mathcal{R}\left(p_{\tau}\right)$ depends continuously on $\tau \in(0,1]$. Hence, for each $\tau$, if we can show $u(z, w)>0$ for $(z, w) \in Q_{p_{\tau}}$ when $\delta_{1}>|z|>\delta_{2}$, where $\delta_{j}(j=1,2)$ are small positive numbers which are independent of the choice of $p_{0}$, then it is clear that after making $\mathcal{P}$ sufficiently small, any point in $N_{\epsilon, p_{0}}^{-}$stays in the positive side of $M$ and $Q_{p_{\tau}}^{c}$ stays in the side of $u<0$. Pick $(z, w) \in Q_{p_{\tau}}$. Then we have

$$
w=\overline{w_{\tau}}+2 i\left(\frac{w+\overline{w_{\tau}}}{2}\right)^{k} h\left(z, \overline{z_{0}}, \frac{w+\overline{w_{\tau}}}{2}\right) .
$$


On the other hand, $\left|t_{0}\right|=s_{0}^{k}\left|h\left(z_{0}, \overline{z_{0}}, s_{0}\right)\right| \lesssim s_{0}^{k}\left|z_{0}\right|$. Hence, when $\epsilon<\delta s_{0}^{k}$ with $\delta$ a sufficiently small number, we get $\operatorname{Im} w=\epsilon \tau+R_{1}$ and $\operatorname{Re}(w)=s_{0}+R_{2}$, where $\left|R_{1}\right|<C^{*} s_{0}^{k}\left|z_{0}\right|$ and $\left|R_{2}\right|<C^{*} s_{0}\left|z_{0}\right|$ with $C^{*}$ a fixed number depending only on $M$ and the initial choice of $\widetilde{\mathcal{P}}$. Therefore, to make $u=-\operatorname{Im}(w)+(\operatorname{Re}(w))^{k} h(z, \bar{z}, s)>$ 0 , it suffices to make

$$
h(z, \bar{z}, s)>\frac{\tau \epsilon+R_{1}}{\left(s_{0}+R_{2}\right)^{k}} .
$$

On the other hand, by shrinking $\mathcal{P}$, we can make $\left|z_{0}\right|$ small; and by making $|z|<\delta_{1}$ with $\delta_{1}$ a small but fixed number, we can make $h(z, \bar{z}, s)>\frac{1}{2}|z|^{2}$. Hence, after shrinking $\mathcal{P}$ and $\delta$ if necessary, we can find another small positive number $\delta_{2}\left(<\delta_{1}\right)$ which is independent of the choice of $p_{0}$, such that when $(z, w) \in Q_{p_{\tau}}$ with $\delta_{1}>$ $|z|>\delta_{2}$, then $u(z, w)>0$. As mentioned before, this implies that the whole line $N_{p_{\tau}, \epsilon}^{-}$stays in the positive side of $M$. The other cases can be verified similarly and the details are left to the reader.

Note that for $p \in \mathcal{P},|u(p)|$ is proportional to the distance of $p$ to $M$. For a small $\delta^{*}>0$, let

$$
U_{\delta^{*}}=\left\{p=(z, w) \in \mathcal{P}: \operatorname{dist}(p, M) \leq \delta^{*}(|\operatorname{Re}(w)|)^{k}\right\} .
$$

Then $U_{\delta^{*}} \backslash M$ has four simply connected components. After shrinking $\delta^{*}$, if necessary, we can find two of them staying in the positive side of $M$. Denote the union of such two components by $U_{\delta^{*}}^{+}$and the union of the other two by $U_{\delta^{*}}^{-}$. It is clear by Lemma 2.2. that we can assume that $U_{\delta^{*}}^{+}$has $M$ near 0 as part of its boundary. Since $\mathcal{R}$ is a diffeomorphic map, by suitably choosing $(\delta \gg) \delta^{* *}>\delta^{*}$, we can assume that $\cup_{p \in U_{\delta^{* *}}^{+}} Q_{p}^{c}$ contains the closure of $U_{\delta^{*}}^{-}$. Also, for each $p \in U_{\delta^{*}}^{-}$there is a continuous family of complex analytic disks attaching to $M$, shrinking down to some point in $M$, whose image contains $p$ (see [E] Proposition 4.3). By applying the Baouendi-Treves approximation theorem [BT2] and the maximum principle of holomorphic functions, it follows that any CR function extends holomorphically to $U_{\delta^{*}}^{-}$. Moreover, for any $\xi \in U_{\delta^{*}}^{-},|h(\xi)| \leq \max _{p \in M \cap \tilde{\mathcal{P}}}|h(p)|$. In what follows, we will fix the above $\delta^{*}$. We will use the notation $O(A)$ for a small neighborhood of $A$ whose size may be different in different contexts. Also, we write $M^{+}=M \cap\{s>0\}$ and $M^{-}=M \cap\{s<0\}$.

Lemma 2.3. Let $M, M^{\prime} \subset \mathbb{C}^{2}$ be Levi non-flat real analytic hypersurfaces, which are of infinite type at 0 and have the $m$-value two at 0 . Let $F$ be a continuous $C R$ map from $M$ into $M^{\prime}$ such that $F(0)=0$. Assume that neither of $F\left(M^{+}\right)$nor $F\left(M^{-}\right)$is contained in a complex analytic variety of dimension 1 . Then $F$ extends to a bounded holomorphic function in $U_{\delta^{*}} \cap O(0)$.

Proof. First, by shrinking $\widetilde{\mathcal{P}}$, we can choose a small neighborhood $\mathcal{P}^{\prime}$ of 0 such that $F(\widetilde{\mathcal{P}} \cap M) \subset \subset \mathcal{P}^{\prime}$. We claim that the nondegeneracy for $F$ as defined in the lemma implies that $F(M \backslash E) \cap E^{\prime}=\emptyset$, where $E^{\prime}$ is the complex hypersurface of infinite 
type points of $M^{\prime}$ near 0 . Indeed, the decomposition of $M$ into local CR orbits (see e.g. [BE]) near 0 is given by $M=M^{+} \cup E \cup M^{-}$and similarly for $M^{\prime}$. Since $F$ is a CR mapping, $F\left(M^{+}\right), F\left(M^{-}\right)$and $F(E)$ are all contained in local CR orbits of $M^{\prime}$. Since $E^{\prime}$ is a local CR orbit in $M^{\prime}$ which is also a 1-dimensional complex variety, and neither $F\left(M^{+}\right)$nor $F\left(M^{-}\right)$, by assumption, is contained a 1-dimensional complex variety, the claim follows. This proves the claim. By the result in [Hu1], $F$ extends holomorphically to a sufficiently small neighborhood of $M^{+} \cup M^{-}$. Since the $m$-value for $M^{\prime}$ is 2 at 0 , one can easily verify that if $\mathcal{P}^{\prime}$ is sufficiently small, $Q_{q_{1}}^{\prime}=Q_{q_{2}}^{\prime}$ with $q_{1}, q_{2} \in \mathcal{P}^{\prime}$ only if $q_{1}=q_{2}$. Here, we use $Q_{q}^{\prime}$ to denote the Segre variety of $M^{\prime}$ associated to $q$.

Now, pick $p_{0}=\left(z_{0}, s_{0}+i t_{0}\right) \in M \cap \mathcal{P}\left(s_{0} \neq 0\right)$ and $\epsilon<\delta\left|s_{0}\right|^{k}$. Here $\delta, \delta^{*}$ are arranged so that $\delta^{*} \ll \delta$. Without loss of generality, let us assume that $N_{p_{0}, \epsilon}^{+}$ stays in the positive side of $M$. (Otherwise, $N_{p_{0}, \epsilon}^{-}$will stay in the positive side of $M$.) We mention that by the proof of Lemma 2.2, any point in the component of $U_{\delta^{*}} \backslash M$, containing $N_{p_{0}, \epsilon}^{+}$, stays also in the positive side of $M$; also, $F$ extends holomorphically to the component containing $N_{p_{0}, \epsilon}^{-}$.

We will show that $F$ admits a holomorphic extension to a neighborhood of $N_{p_{0}, \epsilon}^{+}$, which is uniformly bounded with respect to $p_{0}$. For this purpose, we let

$$
\mathcal{V}_{p_{0}, \epsilon}=\left\{(p, q) \in O\left(N_{p_{0}, \epsilon}^{+}\right) \times \mathcal{P}^{\prime}: F\left(Q_{p}^{c}\right) \subset Q_{q}^{\prime}\right\} .
$$

Then $\mathcal{V}_{p_{0}, \epsilon}$ is a complex analytic variety of dimension two in $O\left(N_{p_{0}, \epsilon}^{+}\right) \times \mathcal{P}^{\prime}$. Write $\pi$ and $\pi^{\prime}$ for the natural projection from $\mathcal{V}_{p_{0}, \epsilon}$ to $O\left(N_{p_{0}, \epsilon}^{+}\right)$and $\mathcal{P}^{\prime}$, respectively. Write $\mathcal{V}^{c}$ for the irreducible component, which contains a neighborhood of $\left(p_{\tau}, F\left(p_{\tau}\right)\right)$ for $\tau \ll 1$. Then, the same argument as in [Hu1, Lemma 3.1] shows that $\pi\left(\mathcal{V}^{c}\right)$ covers $N_{p_{0}, \epsilon}^{+}$. By our choice of $\mathcal{P}^{\prime}$, we may define a map near $N_{p_{0}, \epsilon}^{+}$which assigns to each $p \in O\left(N_{p_{0}, \epsilon}^{+}\right)$the uniquely determined $q$ with $q=\pi^{\prime} \circ \pi^{-1}(p)$. Clearly, this map gives the holomorphic extension of $F$ to a neighborhood of $N_{p_{0}, \epsilon}^{+}$. Moreover, as in the proof of Lemma 3.1 of [Hu1], $\mathcal{R}^{\prime}(q)=F(\xi)$ for some $\xi \in Q_{p}^{c}$. Here $\mathcal{R}^{\prime}$ is the corresponding conjugate function of $M^{\prime}$. As remarked before, we thus conclude from the maximum principle that $|q|$ is uniformly bounded (with respect to $q_{0}$ ). This completes the proof.

Now, let $M, M^{\prime}$ and $F$ be as in Lemma 2.3. For each $(z, w) \in M$, applying the Cauchy estimate to the holomorphic function $F(z, w+\eta)$ in $\eta$ for $|\eta|<\delta^{*}(|\operatorname{Re}(w)|)^{k}$, we get

$$
\left|F_{w}^{\prime}(z, w)\right| \lesssim \frac{\max _{|\eta|=\delta^{*}|s|^{k}}|F(z, w+\eta)|}{|\operatorname{Re}(w)|^{k}} \lesssim \frac{1}{|\operatorname{Re}(w)|^{k}} .
$$

Also, since $|u(z+\xi, w)| \lesssim|\xi| \cdot|\operatorname{Re}(w)|^{k}$, applying Lemma 2.3, we see that $\{F(z+$ $\xi, w)\}$ forms a uniformly bounded holomorphic family in $\xi$ for $|\xi|<\delta_{0}$ with $\delta_{0}$ a fixed but sufficiently small number. Moreover $F(z+\xi, w)$ converges uniformly on compact sets to $F(\xi, 0)$ as $(z, w) \rightarrow 0$. ( Here $(z, w) \in M$ acts as a parameter.) Therefore, it 
follows that for any integer $n, \partial^{n} F / \partial z^{n}$ extends continuously from $(M \backslash E) \cap \mathcal{P}$ to $M \cap \mathcal{P}$. Set

$$
L=\frac{\partial}{\partial z}-\frac{u_{z}^{\prime}}{u_{w}^{\prime}} \frac{\partial}{\partial w}
$$

which is a $(1,0)$-vector field tangent to $M$. Hence, the restriction of $L$ to $M$ is a section of $T^{1,0} M$ and its complex conjugate is a basis for the CR vector fields on $M$. Since $u_{w}^{\prime}=1 / 2+o(1)$ and $\left|u_{z}^{\prime}\right| \lesssim|z||\operatorname{Re}(w)|^{k}$, the function $L(F)=F_{z}^{\prime}-u_{z}^{\prime} F_{w}^{\prime} / u_{w}^{\prime}$,

which as mentioned above is real analytic over $M \backslash E$ in view of the result in [Hu1], is bounded in $M \backslash E$ near 0 and $\lim _{p \rightarrow 0} L(F)(p)=F_{z}^{\prime}(0)$, for $p \in M \backslash E$. Now, for $p_{0}$ sufficiently close to 0 such that $m\left(p_{0}\right)=m^{\prime}\left(F\left(p_{0}\right)\right)=2$, we can also choose normal coordinates (as given by Lemma 2.1) for $M$ and $M^{\prime}$ near $p_{0}$ and $F\left(p_{0}\right)$, respectively. By repeating the same analysis as above in the new coordinates near $p_{0}$, we conclude that there exists a real-analytic nonvanishing $(1,0)$-vector field $\widetilde{L}$ tangent to $M$ near $p_{0}$ such that $\widetilde{L}(F)$ has a limit at $p_{0}$. Since the dimension of $T^{1,0} M$ is one, $\widetilde{L}=a(z, w) L$, for some real-analytic nonvanishing function $a(z, w)$, we deduce that $L(F)$ has a limit at $p_{0}$ and $L(F)$ is continuous in a neighborhood of 0 in $M$. Summarizing the above, we obtain the following lemma, which is crucial for the proof of Theorem 2.

Lemma 2.4. Let $M, M^{\prime}$ and $F$ be as in Lemma 2.3. Then $L(F)=F_{z}^{\prime}-u_{z}^{\prime} F_{w}^{\prime} / u_{w}^{\prime}$ extends continuously from $M \backslash E$ to a neighborhood of 0 in $M$.

\section{Proof of the Theorem 2}

With the aid of Lemma 2.4, we can now complete the proof of Theorem 2 as follows.

Let $M, M^{\prime}$ be of infinite type at 0 , and let $F$ be a continuous CR map from $M$ into $M^{\prime}$ with $F(0)=0$ which extends holomorphically to one side $D$ of $M$ such that $F$ is continuous over $D \cup M$. Let $\delta \subset M$ be a real analytic totally real submanifold of real dimension 2 passing through $p=0$ such that $\delta \cap E$ is a real analytic curve near 0 . In what follows, we shall assume, without loss of generality, that $E$ is still $\{w=0\}$ but $\delta=\mathbb{R}^{2} \cap O(0)$. Moreover, we assume that the positive $\operatorname{Im} w$-axis is the outward normal direction of $M$ along $\&$ near 0 with respect to the $D$-side. Observe that in these coordinates the defining equation for $M$ is in general not of the form given by Lemma 2.1. For a small $\widetilde{\delta}>0$, write $\mathcal{W}^{ \pm}=\{(z, w): \pm \widetilde{\delta} \operatorname{Im} w>|\operatorname{Im} z|\}$. Then $\mathcal{W}^{+} \subset O(0) \backslash D \cup M$ and $(\bar{z}, \bar{w}) \in D$ for $(z, w) \in \mathcal{W}^{+}$near the origin. Let $\beta^{*}(\in \mathbb{R}) \approx 0, \beta=\left(\beta_{1}, \beta_{2}\right) \in \mathbb{R}^{2}$ with $\beta_{2} \approx 1$ and $\beta_{1} \approx 0$. Write $\Delta_{\epsilon}=\{\zeta \in \mathbb{C}:$ $|\zeta|<\epsilon\}, \Delta_{\epsilon}{ }^{+}=\left\{\zeta \in \Delta_{\epsilon}: \operatorname{Im} \zeta>0\right\}$. For $\epsilon$ sufficiently small, we define $\psi_{\beta, \beta^{*}}$ by $\zeta \longrightarrow\left(\beta_{1} \zeta+\beta^{*}, \beta_{2} \zeta\right)$. Then it is clear that $\psi_{\beta, \beta^{*}}\left(\Delta \backslash\left(\Delta_{\epsilon}^{+} \cup(-\epsilon, \epsilon)\right) \subset D\right.$. In what follows, we will also write $(\operatorname{Re}(z), \operatorname{Re}(w))=X$.

We choose the normal coordinates for $M^{\prime}$ so that it is defined near 0 by an equation of the form $t=s^{k^{\prime}} \chi(z, \bar{z}, s)$ with $\chi(z, 0, s)=\chi(0, \bar{z}, s)=0$ and $k^{\prime}>0$. Write 
$F=(f, g)$. We shall refer to $g$ as the normal component of $F$. It is clear that $g(z, 0)=0$ since $F(E) \subset E^{\prime}=\{w=0\}$. On the other hand, by condition (i) in Theorem 2 and the remark at the beginning of the proof of Lemma 2.3, $g \neq 0$ for $(z, w) \in M \backslash E$ since $F(M \backslash E) \cap E^{\prime}=\emptyset$. The following is another crucial lemma for the proof of Theorem 2 .

Proposition 3.1. There is a positive integer $n$ and a point $p_{0}=\left(\beta_{0}, 0\right) \in \& \cap E$ near 0 such that for any positive integer $m, 1 \overline{g^{m}(X)}$, initially defined over $\$ \backslash E$, has a unique holomorphic extension $h_{m}(z, w)$ to $W^{+} \cap O\left(p_{0}\right)$. Moreover, $\left|h_{m}(z, w)\right| \lesssim 1 /|\operatorname{Im} w|^{N}$

for some integer $N$ depending only on $m$, and $h_{m}(z, w)$ has the limit $1 / \overline{g^{m}(X)}$ as $(z, w) \rightarrow X \in s \backslash E$.

Proposition 3.1 readily follows from Proposition 3.2 of [HMM] when $F$ is assumed to be Hölder continuous. (In this case, $h_{m}$ was actually proved to be Hölder continuous over $\left.\overline{\mathcal{W}^{+}} \cap O\left(p_{0}\right)[\mathbf{H M M}]\right)$. In the present context, the proof can be carried out by a similar argument. Let us start with the following Riemann-Hilbert problem. We shall use the notation $\Delta_{r}^{+}=\{z \in \mathbb{C}:|z|<r, \operatorname{Im}(z)>0\}$, and $\Delta^{+}=\Delta_{1}^{+}$.

Lemma 3.2. Let $\phi(z, \tau)=u(z, \tau)+i v(z, \tau)$ be a nonconstant holomorphic function of $z$ in $\Delta^{+}$, which is continuous over $\overline{\Delta^{+}} \times O\left(\tau_{0}\right)$, where $O\left(\tau_{0}\right)$ is a small neighborhood of $\tau_{0}$ in the parameter $\tau$-space. Suppose that for $x \in(-1,1), v(x, \tau)=$ $u(x, \tau) P(x, \tau)$ with $P(x, \tau) \in C\left((-1,1) \times O\left(\tau_{0}\right)\right)$. Then there exists a non-negative integer $n$, a small $\epsilon_{0}>0$, and a positive constant $C_{0}$ such that $\phi(z, \tau)=z^{n} \phi^{*}(z, \tau)$, where $\phi^{*}(z, \tau)$ is a holomorphic function of $z$ in $\Delta_{\epsilon_{0}}^{+}$and

$$
\sup _{0 \leq y<\epsilon_{0}} \int_{-\epsilon_{0}}^{\epsilon_{0}}\left|\phi^{*}(x+i y, \tau)\right| \mathrm{d} x<C_{0}, \quad \sup _{0 \leq y<\epsilon_{0}} \int_{-\epsilon_{0}}^{\epsilon_{0}}\left|\frac{1}{\phi^{*}(x+i y, \tau)}\right| \mathrm{d} x<C_{0}
$$

for $\tau \in O\left(\tau_{0}^{*}\right)$, where $\tau_{0}^{*}$ is some point close to $\tau_{0}$.

Proof. Write $\phi^{+}(z, \tau)=\phi(z, \tau)$ and $\phi^{-}(z, \tau)=\overline{\phi(\bar{z}, \tau)}$ for $z \in \overline{\Delta^{-}}=\{z \in \mathbb{C}:$ $|z| \leq 1, \operatorname{Im}(z)<0\}$. Then for $x \in(-1,1)$, one has

$$
v(x, \tau)=\frac{1}{2 i}\left(\phi^{+}(x, \tau)-\phi^{-}(x, \tau)\right), \quad u(x, \tau)=\frac{1}{2}\left(\phi^{+}(x, \tau)+\phi^{-}(x, \tau)\right) .
$$

Substituting this into the equation $v=u P(x, \tau)$, we obtain

$$
\frac{1+i P(x, \tau)}{1-i P(x, \tau)} \phi^{-}(x, \tau)=\phi^{+}(x, \tau) .
$$

Now, let $\epsilon_{0}$ be a sufficiently small positive integer and $\eta(x)$ a smooth cutoff function supported in $\left(-3 \epsilon_{0}, 3 \epsilon_{0}\right)$ with $\eta(x) \equiv 1$ for $|x|<2 \epsilon_{0} \ll 1 / 2$. Consider the following Cauchy integral

$$
\Gamma(z, \tau)=\frac{1}{2 \pi i} \int_{-\infty}^{+\infty}\left(\eta(\xi) \log \frac{1+i P(\xi, \tau)}{1-i P(\xi, \tau)}\right) \frac{\mathrm{d} \xi}{\xi-z}
$$


By the Plemelj formula, we have

$$
\mathrm{e}^{\Gamma^{+}(x, \tau)-\Gamma^{-}(x, \tau)}=\left(\frac{1+i P(x, \tau)}{1-i P(x, \tau)}\right)^{\eta(x)}
$$

for almost every $x \in(-1,1)$. Here for $z \notin(-1,1), \Gamma^{ \pm}(z, \tau)$ is defined to be the value of $\Gamma(z, \tau)$ for $z \in \Delta^{ \pm}$, respectively; and the value $\Gamma^{ \pm}(x, \tau)$ on $x \in(-1,1)$ is understood as the non-tangential boundary limit of $\Gamma^{ \pm}(z, \tau)$ as $z\left(\in \Delta^{ \pm}\right) \rightarrow x$, respectively. Moreover, if we denote by $\mathscr{H}_{r}$ the Hilbert transform on $\mathbb{R}$ defined by $\mathscr{H}_{r}(u)(x)=\frac{1}{\pi} \int_{-\infty}^{+\infty} \frac{u(t)}{t-x} d t$, then

$$
\Gamma^{ \pm}(x, \tau)=\frac{1}{2}\left( \pm u_{\tau}(x)-i \mathscr{H}_{r}\left(u_{\tau}\right)(x)\right),
$$

where

$$
u_{\tau}(\xi)=\eta(\xi) \log \frac{1+i P(\xi, \tau)}{1-i P(\xi, \tau)} .
$$

Recall that on the unit circle, the Hilbert transform $\mathscr{H}$, which maps $u$ to its harmonic conjugate vanishing at 0 , and the Cauchy singular integral operator $\delta$ are related by the formula

$$
\mathscr{H}(\phi)=i \S(\phi)-\frac{1}{2 \pi} \int_{|\xi|=1} \frac{\phi(\xi)}{\xi} \mathrm{d} \xi .
$$

Also, by a classical result on the Hilbert transform on the unit circle (see [Z], Theorem 2.11, p. 254, and its proof on p. 257 of $[\mathbf{Z}])$,

$$
\int_{0}^{2 \pi} \mathrm{e}^{|\mathcal{H}(\phi)|} \mathrm{d} \theta<\frac{4 \pi}{\cos (1)}
$$

for any continuous function $\phi$ with $|\phi| \leq 1$. Hence, after making use of a linear fractional transformation and basic properties of $H^{1}$-spaces, we can find some fixed constant $C^{*}$ such that for $\tau \approx \tau_{0}$

$$
\sup _{0 \leq y<\epsilon_{0}} \int_{-\epsilon_{0}}^{\epsilon_{0}} \mathrm{e}^{|\Gamma(x \pm i y, \tau)|} \mathrm{d} x<C^{*} .
$$

Since $\phi^{+}(x, \tau) \mathrm{e}^{-\Gamma^{+}(x, \tau)}=\phi^{-}(x, \tau) \mathrm{e}^{-\Gamma^{-}(x, \tau)}$, it follows that $\phi^{+}(x, \tau) \mathrm{e}^{-\Gamma^{+}(x, \tau)}$ extends holomorphically in $z$ to the disk $\Delta_{\epsilon_{0}}$. Denote its extension by $\widetilde{\phi}(z, \tau)$. Then (3.1) can also be used to show that $\{\widetilde{\phi}(z, \tau)\}$ is a normal family with parameter $\tau\left(\approx \tau_{0}\right)$ for $|z|<\epsilon_{0}$. Meanwhile, since $\phi^{ \pm}(z, \tau)$ and $\mathrm{e}^{\Gamma^{ \pm}(z, \tau)}$ depend continuously on $\tau$ when $z \notin(-1,1)$, we conclude that $\lim _{\tau \rightarrow \tau^{*}} \widetilde{\phi}(z, \tau)=\widetilde{\phi}\left(z, \tau^{*}\right)$ for any $\tau^{*} \in O\left(\tau_{0}\right)$. Write $\widetilde{\phi}(z, \tau)=z^{k_{\tau}} \widetilde{\phi}^{*}(z, \tau)$ with $\widetilde{\phi}^{*}(0, \tau) \neq 0$. By the Hurwitz theorem, $k_{\tau}$ is upper semi-continuous in $\tau$. Now, since $k_{\tau}$ only takes integer value, we can find a point $\tau_{0}^{*}$ sufficiently close to $\tau_{0}$ and an integer $n$, such that for $\tau \approx \tau_{0}^{*}, k_{\tau}=n$ and $\widetilde{\phi}^{*}(\xi, \tau)$ has no zeros over $\Delta_{\epsilon_{0}} \backslash\{0\}$ (after shrinking $\epsilon_{0}$ (if necessary). The desired $\phi^{*}(z, \tau)$ is 
obtained by $\phi^{*}(z, \tau)=\widetilde{\phi}^{*}(z, \tau) \mathrm{e}^{\Gamma^{+}(z, t)}$. Moreover, by (3.1), we obtain

$$
\sup _{0 \leq y<\epsilon_{0}} \int_{-\epsilon_{0}}^{\epsilon_{0}}\left|\phi^{*}(x+i y, \tau)\right| \mathrm{d} x, \quad \sup _{0 \leq y<\epsilon_{0}} \int_{-\epsilon_{0}}^{\epsilon_{0}} \frac{1}{\left|\phi^{*}(x+i y, \tau)\right|} \mathrm{d} x<C_{0}
$$

for some fixed constant $C_{0}$ independent of $\tau \approx \tau_{0}^{*}$. This completes the proof.

Proof of Proposition 3.1. Assume, without loss of generality, that $g\left(\beta^{*}, t\right) \not \equiv 0$ for $\beta^{*}(\in \mathbb{R}) \approx 0$ and $t<0$. Write $\tau=\left(\beta_{1}, \beta_{2}, \beta^{*}\right) \approx(0,1,0)$, and write $g_{\tau}=$ $g \circ \psi_{\tau}(\bar{\zeta})$, where we recall that $\psi_{\tau}(\zeta)=\left(\beta_{1} \zeta+\beta^{*}, \beta_{2} \zeta\right)$. Then $g_{\tau}(\zeta)$ depends continuously on $\tau=\left(\beta_{1}, \beta_{2}, \beta^{*}\right)$ and $\zeta \in(-\epsilon, \epsilon)$. Also, it extends holomorphically to $\Delta_{\epsilon}^{+}$in $\zeta$. Note that for $\zeta \in(-\epsilon, \epsilon), \psi_{\tau}(\zeta) \in M$ and, hence, $\operatorname{Im}\left(g_{\tau}(\zeta)\right)=$ $-\left(\operatorname{Re}\left(g_{\tau}(\zeta)\right)\right)^{m} \chi\left(f \circ \psi_{\tau}, \overline{f \circ \psi_{\tau}}, \operatorname{Re}\left(g_{\tau}(\zeta)\right)\right.$. Applying Lemma 3.2, we obtain the existence of a parameter $\tau_{0}=\left(\beta_{1}^{0}, \beta_{2}^{0}, \beta_{0}^{*}\right)$ and an integer $n$ such that for the sufficiently small $\epsilon_{0}<\epsilon$ and $\tau \approx \tau_{0}, g_{\tau}(\zeta)=\zeta^{n} g_{\tau}^{*}(\zeta)$ with $g_{\tau}^{*}$ holomorphic in $\Delta_{\epsilon_{0}}^{+}$and

$$
\sup _{0 \leq y \leq \epsilon_{0}} \int_{-\epsilon_{0}}^{\epsilon_{0}}\left|g_{\tau}^{*}(x+i y)\right| \mathrm{d} x, \quad \sup _{0<y \leq \epsilon_{0}} \int_{-\epsilon_{0}}^{\epsilon_{0}} \frac{1}{\left|g_{\tau}^{*}(x+i y)\right|} \mathrm{d} x
$$

uniformly bounded with respect to $\tau\left(\approx \tau_{0}\right)$. Hence, the Cauchy formula

$$
1 / g_{\tau}^{*}(\zeta)=\frac{1}{2 \pi i} \int_{\partial \Delta_{\epsilon_{0}}^{+}} \frac{1}{g_{\tau}^{*}(z)(z-\zeta)} \mathrm{d} z, \quad \zeta \in \Delta_{\epsilon_{0}}^{+},
$$

and the above estimates imply that for $\zeta \in \Delta^{+} \cap O(0)$

$$
\frac{1}{\left|g_{\tau}^{*}(\zeta)\right|} \lesssim \frac{1}{|\operatorname{Im}(\zeta)|}
$$

uniformly in $\tau\left(\approx \tau_{0}\right)$.

Denote by $z_{0}$ the point $\left(\beta_{0}^{*}, 0\right)$. Shrinking $\tilde{\delta}$ in the definition of $\mathcal{W}^{+}$if necessary, then the union of the image of the disks $(z, w)=\psi_{\tau}(\zeta)=\left(\beta_{1} \zeta+\beta^{*}, \beta_{2} \zeta\right)$, for $\tau \approx \tau_{0}, \zeta \in\{\zeta, \operatorname{Im}(\zeta)>0\} \cap O(0)$ fills the set $\mathcal{W}^{+} \cap O\left(z_{0}\right)$. Hence, by setting $(z, w)=\overline{\psi_{\tau}(\bar{\zeta})}$ and using $\operatorname{Im} w=\beta_{2} \operatorname{Im} \zeta$,

$$
h_{1}(z, w)=\frac{1}{\overline{g((\bar{z}, \bar{w}))}}=\frac{1}{|\zeta|^{n}\left|g_{\tau}^{*}(\zeta)\right|} \lesssim \frac{1}{|\operatorname{Im} w|^{n+1}}
$$

for $(z, w) \in \mathcal{W}^{+} \cap O\left(z_{0}\right)$. Since $g \not \equiv 0$, we have $F(M \backslash E) \subset M^{\prime} \backslash E^{\prime}$ as already pointed out in the proof of Lemma 2.3. Thus $F \in C^{\omega}(M \backslash E)$ by [Hu1]. The proof of Proposition 3.1 now follows.

Proof of Theorem 2. The fact that the 1-dimensional complex analytic variety $E$ through 0 is contained in the real hypersurface $M \subset \mathbb{C}^{2}$ implies that $E$ is a complex (nonsingular) curve, as is well known, and the restriction of $F$ to $E$ is a holomorphic mapping $E \rightarrow M^{\prime} \subset \mathbb{C}^{2}$. Since $\left.F\right|_{E}$ is not constant, it is locally finite-to-one and the image $F(E)=E^{\prime} \subset M^{\prime}$ is a 1-dimensional complex analytic variety through 0 . Hence, $E^{\prime}$ is also a complex (nonsingular) curve. To complete the proof of Theorem 2, 
in view of the Hanges-Treves propagation theorem [HT], we need only show that $F$ extends holomorphically to a neighborhood of some point in $E$. Thus, in what follows, we reserve the right to shift the origin in $M$ to any nearby point $p \in E \subset M$. By applying Lemma 2.2, we may assume, without loss of generality, that $m(0)=m^{\prime}(0)=2$, $F(0)=0$, and $\left.F\right|_{E}$ is locally invertible near 0 . Shifting again to a nearby point if necessary, we may also assume that the conclusion of Proposition 3.1 holds with $p_{0}=0$.

Let $u=t+o(|z, w|)$ be a real analytic defining function of $M$, and let $\&$ and its wedges $W^{ \pm}$be chosen as before. Recall the $(1,0)$-vector field

$$
L=\frac{\partial}{\partial z}-\frac{u_{z}}{u_{w}} \frac{\partial}{\partial w}
$$

which is tangent to $M$. We also use $L$ for the restriction of this vector field to $M$. By Lemma $2.4, L(F)$ is be continuous over $M$ and $L(f)(0)=f_{z}^{\prime}(0) \neq 0$. Also, recall that $M^{\prime}$ is defined by $t=s^{k} \chi(z, \bar{z}, s)$ with $\chi(z, \bar{z}, 0)= \pm|z|^{2}+o(|z|), \chi(z, 0, s)=0$. Since $f(M) \subset M^{\prime}$, we obtain $\operatorname{Im}(g(z))=(\operatorname{Re}(g(z)))^{m} \chi(f(z), \overline{f(z)}, \operatorname{Re}(g(z)))$ for $z \in M$. By applying the implicit function theorem, we obtain the following functional equation

$$
\overline{g(z)}=g(z)+(g(z))^{m} G(f(z), \overline{f(z)}, g(z)),
$$

for $z \in M$, where $G(a, \bar{a}, 0)$ does not have any harmonic terms and $G(a, b, 0)=$ $c_{0} a b+o(a b)$ with $c_{0} \neq 0$.

Now, applying $\bar{L}$ to (3.2), we obtain

$$
\overline{L g}=(g(z, w))^{k} \frac{\partial G}{\partial b}(f(z, w), \overline{f(z, w)}, g(z, w)) \overline{L f} .
$$

Since $\overline{L(F)}$ is continuous along $\&$, and $\overline{L(F)}$ extends holomorphically to the wedge $W^{+}$and blows up at most polynomially when approaching the edge $\delta$, we conclude that $\overline{L(F)}$ has a unique holomorphic extension to $\mathcal{W}^{+}$, which is continuous up to $\&$ (see [BJT] or [BE] for instance). In particular, we see that $1 / \overline{L(f)}$ has an extension which is holomorphic in $\mathfrak{W}^{+}$and continuous in $\overline{W^{+}} \cap O(0)$. The reader should be warned that the holomorphic extension of $L(F)$ from $\&$ into $W^{-}$need not coincide with the $\mathbb{C}^{2}$-valued function obtained by applying the $(1,0)$-vector field $L$ to the holomorphic mapping $F$ in $\mathcal{W}^{-}$.

Next, since

$$
g(z, w)=\overline{g(z, w)}+(\overline{g(z, w)})^{k} \overline{G(f(z, w), \overline{f(z, w)}, g(z, w))},
$$

we obtain

$$
\frac{\overline{L g}}{\overline{L(f)} \overline{g^{k}}}=\left(1+(\bar{g})^{k-1} \overline{G(f, \bar{f}, g)}\right)^{k} \frac{\partial G}{\partial b}(f, \bar{f}, g) \text {. }
$$

By the discussion above and Proposition 3.1, $\overline{L g} /\left(\overline{L(f)} \overline{g^{k}}\right)$ has a unique holomorphic extension $\chi(z, w)$ which is holomorphic in $\mathcal{W}^{+} \cap O(0)$ and blows up at most polyno- 
mially when $(z, w) \rightarrow \S$. Hence, $\chi(z, w)$ has the limit, in the sense of distributions,

$$
\left(1+(\overline{g(z, w)})^{k-1} \overline{G(f(z), \overline{f(z)}, g(z))}\right)^{k} \frac{\partial G}{\partial b}(f(z, w), \overline{f(z, w)}, g(z, w))
$$

along $\&$ and, since (3.6) is continuous over $\&$, we conclude that $\chi(z, w) \in \operatorname{Hol}\left(\mathcal{W}^{+}\right) \cap$ $C\left(\overline{\mathfrak{W}^{+}} \cap O(0)\right)$.

Since $\partial G^{2} / \partial a \partial b(0) \neq 0$, by applying the implicit function theorem to (3.5) in which we have substituted (3.4), we deduce that near $0 \in \delta$,

$$
f(X)=\sigma_{1}(\overline{f(X)}, \overline{g(X)}, \chi(X))
$$

where $X:=\operatorname{Re}(z, w)$ and $\sigma_{1}$ is some function holomorphic in its argument. By the formula (3.4), we can find a $\sigma_{2}$ holomorphic in its argument such that for $X \in \&$ near $0, g(X)=\sigma_{2}(\overline{f(X)}, \overline{g(X)}, \chi(X))$.

Since $F(X)=(f(X), g(X))$ extends holomorphically to $\mathcal{W}^{-}$and, for $j=1,2$, $\sigma_{j}(\overline{f(X)}, \overline{g(X)}, \chi)$ extend holomorphically to $\mathfrak{W}^{+}$, it follows that $F$ extends holomorphically to an open neighborhood of 0 by the classical edge of the wedge theorem, As mentioned above, by the Hanges-Treves theorem, $F$ extends holomorphically to a neighborhood of $E$. This completes the proof of Theorem 2 .

Proof of Theorem 1. Let $p_{0} \in M$ be a point of finite type, i.e., one through which $M$ does not contain any nontrivial complex curves. By the same argument used in the beginning of the proof of Lemma 2.3 and condition (i), we conclude that $F\left(p_{0}\right)$ is of finite type in $M$. The holomorphic extension of $F$ across $p_{0}$ follows from the result in [Hu1]. If $p_{0} \in M$ is a point of infinite type, then the holomorphic extension across $p_{0}$ follows from Theorem 2 .

\section{References}

[BBR] M. S. Baouendi, S. Bell and L. P. Rothschild, Mappings of three-dimensional CR manifolds and their holomorphic extension, Duke Math. J. 56 (1988), 503-530.

[BE] M. S. Baouendi, P. Ebenfelt and L. P. Rothschild, Real submanifolds in complex spaces and their mappings, Princeton Math. Ser. 47, Princeton Univ. Press, 1999.

[BHR] M. S. Baouendi, X. Huang and L. P. Rothschild, Regularity of CR mappings between algebraic hypersurfaces, Invent. Math. 125 (1996), 13-36.

[BJT] M. S. Baouendi, H. Jacobowitz and F. Treves, On the analyticity of CR mappings, Ann. of Math. 122 (1985), 365-400.

[BR1] M. S. Baouendi and L.P. Rothschild, Germs of CR maps between real analytic hypersurfaces, Invent. Math. 93 (1988) 481-500.

[BR2] M. S. Baouendi and L. P. Rothschild, A general reflection principle in $\mathbb{C}^{2}$, J. Funct. Anal. 99 (1991), 409-442.

[BT1] M. S. Baouendi and F. Treves, About the holomorphic extension of CR functions on real hypersurfaces in complex space, Duke Math. J. 51 (1984), 77-107. 
[BT2] M. S. Baouendi and F. Treves, A property of the functions and distributions annihilated by a locally integrable system of complex vector fields, Ann. of Math. 113 (1981), 387-421.

[DF] K. Diederich and J.-E. Fornaess, Proper holomorphic mappings between real-analytic pseudoconvex domains in $\mathbb{C}^{n}$, Math. Ann. 282 (1988), 681-700.

[E] P. Ebenfelt, On the unique continuation problem for CR mappings into nonminimal hypersurfaces, J. Geom. Anal. 6 (1996), 385-405.

[HT] N. Hanges and F. Treves, Propagation of holomorphic extendability of CR functions, Math. Ann. 263 (1983), 157-177.

[Hu1] X. Huang, Schwarz reflection principle in complex space of dimension two, Comm. Partial Differential Equations 21 (1996), 1781-1828.

[Hu2] -, On some problems in several complex variables and CR geometry, Proceedings of ICCM (S. T. Yau, ed.), AMS/IP Stud. Adv. Math. 20, 2001, 383-396.

[Hu3] X. Huang, Regularity of holomorphic correspondences and applications to the mapping problem, preprint, circulated in August 1995.

[HMM] X. Huang, J. Merker and F. Meylan, Mappings between degenerate real analytic hypersurfaces in $\mathbb{C}^{n}$, in: Analysis, Geometry, Number Theory: The Mathematics of Leon Ehrenpreis (E. I. Grinberg et al., eds.), Contemp. Math. 251, Amer. Math. Soc., Providence, RI, 2000, 321-338.

[Me] F. Meylan, A reflection principle in complex space for a class of hypersurfaces and mappings, Pacific J. Math. 169 (1995), 135-160.

[Tr] J. M. Trepreau, Sur le prolongement holomorphe des fonctions CR définies sur une hypersurface réelle de classe $C^{2}$ dans $\mathbb{C}^{n}$, Invent. Math. 83 (1986), 583-592.

[Tu] A. E. Tumanov, Extension of CR functions into a wdge from a manifold of finite type, Mat. Sb. 136 (1988), 128-139 = Math. USSR-Sb. 64 (1989), 129-140.

[We1] S. Webster, On the mapping problem for algebraic real hypersurfaces, Invent. Math. 43 (1977), 53-68.

[We2] S. Webster, Biholomorphic mappings and the Bergmann kernels off diagonal, Invent. Math. 51 (1979), 155-169.

[Z] A. Zygmund, Trignometric Series, Vol. I, Cambridge University Press, 2nd Edition, Cambridge 1968.

\footnotetext{
Department of Mathematics Royal Institute of Technology

10044 Stockholm, Sweden

ebenfelt@math.kth.se

Department of Mathematics

Rutgers University

New Brunswick, NJ 08903, U.S.A.

huangx@math . rutgers . edu
} 